whatever, had been "lost" for the "Free World" it was time to hound the Owen Lattimores.

Equally, Mr Hollander refuses to accept that, whatever their quirks and absurdities, the inclination of intellectual propagandists towards presumed cradles of a better world and their aversion against the napalm-throwing Democrats of Viet Nam fame arises from a real malaise. Global inequalities and the terror used to preserve them are no figments concocted by idle assistant lecturers. The blind admiration of some for false gods may be wrongheaded, but the need for new cures is not obviated by such diagnosis.

By way of conclusion, Mr Hollander advocates that we be resolutely anti-utopian and commends and end to all "isms" (p 432). He even appears to fear for the survival of democracy if too many "Morning Deluges" should sweep Western lands ( $p$ 434). We must accept with humble respect that life under Soviet-style régimes may inspire such an attitude. It is, however, not a way out. The terms of trade for poor nations will not improve if Western intellectuals deny themselves, or are denied (?), to face the privileges on which their home countries thrive on an international scale. Also, as we have seen, the community of blind and purblind purveyors of intelligence on distant places extends well into chancelleries and cabinets. The problem is much larger, and having enjoyed Mr Leys's elegant polemical sketches one is left exhausted and not much wiser after Mr Hollander's tome of footnoted spadework.

Wolfgang Kessler

\title{
Frieder Schmelz
}

\section{Paraguay im 19. Jahrhundert}

Ein früher Fall dissoziativer Entwicklung

Esprint-Verlag, Heidelberg, 1981, Heidelberger Dritte Welt Studien, Band 3, DM 19,80

Die von F. Schmelz vorgelegte Schrift verfolgt einen doppelten Zweck. Sie interpretiert die teils aufgezwungene, teils selbstgewählte Isolation Paraguays zwischen 1811 und dem Krieg der Trippelallianz 1865-1870 als eine Art naturwüchsige Dissoziationsstrategie und untersucht, ob und inwieweit die nach dem Senghaas'schen Ansatz zu erwartende autozentrierte Entwicklung eingesetzt hat. Sie kann dabei zeigen, daß im Falle Paraguays die - partielle - Dissoziation zwar nicht zu einer Industrialisierung europäischen Musters geführt hat, wohl aber zu einer breit gestreuten Produktionspalette und vor allem zu dem, was man heute die Erfüllung der Grundbedürfnisse nennen würde. Im Vergleich zu den freihandelsorientierten und von Bürgerkriegen zerrütteten Nachbarn galt Paraguay als Hort der Stabilität und Prosperität.

Dennoch aber hatte das Land bei den zeitgenössischen Beobachtern und lange Zeit auch in der Geschichtsschreibung einen ausgesprochen schlechten Ruf: Vor allem das Regime Dr. Francias (1811-1840) galt als blutige Tyrannei, die Bevölkerung als versklavt und 
unterdrückt. Der Autor zeigt im zweiten Teil seiner Arbeit, wie die zeitgenössischen Besucher des Landes in einer Weise von dem Freihandelsdogma ihrer Zeit geprägt waren, daß sie eine alternative Wirtschaftspolitik nur als das Werk einer schlechten Regierung interpretieren und den damit verbundenen Wohlfahrtseffekt übersehen mußten. Zu dieser Sichtweise mag auch beigetragen haben, daß man im rückständigen Paraguay gegenüber den Sendboten der europäischen Zivilisation oft den nötigen Respekt vermissen ließ.

Auch nach dem Erscheinen der sehr umfangreichen und detaillierten Paraguay-Studie von John Hoyt Williams (1979), die von Schmelz nicht mehr berücksichtigt werden konnte, lohnt sich die Lektüre dieser kleinen Untersuchung, da sie, anders als bei Williams, primär von entwicklungstheoretischen Fragestellungen ausgeht, und außerdem zeigt, wie im 19. Jahrhundert öffentliche Meinung gemacht wurde, die dann bis heute die Interpretation von abweichendem Entwicklungsṿerhalten prägen konnte.

Andreas Boeckh 